\title{
Profesionales en Colombia: ¿lectores críticos? Un estudio sobre la competencia de lectoescritura al interior de las profesiones más influyentes en Colombia
}

\author{
Colombian Undergraduates: Critical Readers? \\ A Study on Literacy Skills within the Most Demanded \\ Undergraduates in Colombia's Labor Market
}

Germán Acero Amaya, Lucas Daniel Aguirre, Diego Fernández Rodríguez, Jorge Andrés Moya, Yoan Romero Ramírez y Nicolás Sierra Rojas"

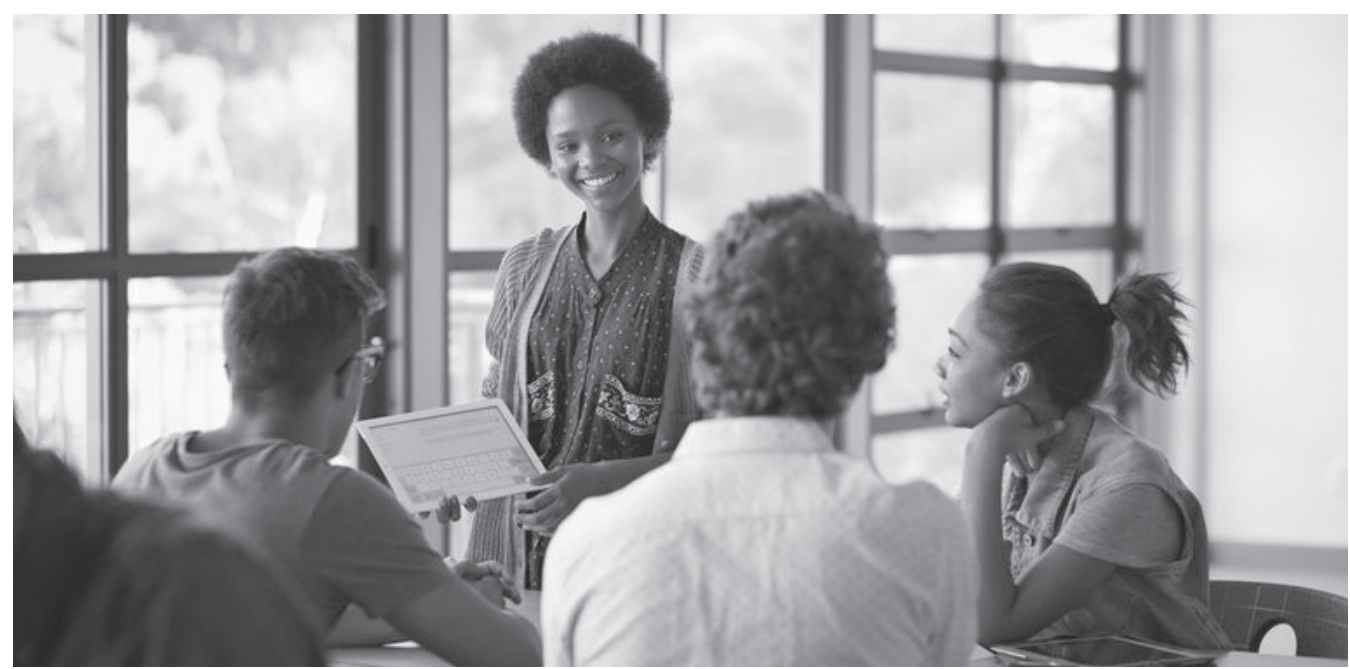

\section{Resumen}

Este estudio busca arrojar indicios sobre cómo fomenta el mercado laboral colombiano la formación en la competencia de lectoescritura de los profesionales que egresan cada año de las universidades de Colombia. Para esto, dirigimos nuestras miradas a la Universidad Nacional de Colombia con el objetivo de contrastar aquellos

Citar este artículo como: Acero Amaya, G., Aguirre, L. D., Fernández Rodríguez, D., Moya, J. A., Romero Ramírez, Y. y Sierra Rojas, N. (2017). Profesionales en Colombia: ¿lectores críticos? Un estudio sobre la competencia de lectoescritura al interior de las profesiones más influyentes en Colombia. Revista Papeles, 9(17), 96-106.

Fecha de recibido: marzo 15 de 2017.

Fecha de aceptación: mayo 30 de 2017.

* Estudiantes de la Facultad de Economía de la Universidad Nacional. Este trabajo fue producto de la investigación al interior del seminario de Metodología de la Investigación, bajo la dirección del profesor Enrique Ferrer-Corredor. Correos: gaceroa@unal.edu.co, ldaguirre@unal.edu.co, dffernandezr@unal.edu.co, jmoyaa@ unal.edu.co, yromeror@unal.edu.co,nisierraro@unal.edu.co 
programas con mejor desempeño en las pruebas de estado, contra los que evidencian una mayor acogida laboral al terminar sus estudios. Encontrando así que, a pesar de lo crucial que es la lectura crítica para el desenvolvimiento del individuo, esto no se manifiesta en el panorama laboral.

Palabras clave: mercado laboral, profesionales colombianos, lectoescritura, pruebas de estado, salarios, competencias, ingreso, empleabilidad.

\begin{abstract}
The purpose of this study is to shed clues on how the Colombian labor market promotes higher reading-writing skills by the undergraduates that finish their studies every year at Colombian universities. Thus, we turn to the Colombian National University looking to make a contrast between the undergraduate programs with a better performance at the standardized public tests, and those programs whose students experience a bigger welcome by the labor market once they finish their studies. Thereby, we find that, in spite of how crucial are reading-writing skills on an individual's development, this is not as evident in the working world.
\end{abstract}

Keywords: Labor Market, Colombian undergraduates, Literacy, Standardized Public Tests, Wages, Skills, Income, Employability.

\title{
Introducción
}

Este trabajo de investigación hace una revisión de la habilidad lectoescritora, siendo esta importante para el buen desarrollo del profesional colombiano, además de necesaria para su desenvolvimiento eficiente en el ámbito laboral; su carencia, por el contrario, llevará a que él mismo sufra falencias en el momento de desarrollar procesos, ya sean pedagógicos o comunicativos.

Se empezará hablando de la lectoescritura como la principal herramienta del profesional, condición contrariada por una problemática generalizada desde un nivel académico más bajo que el del profesional: los estudiantes llegarán a niveles de educación superior con yerros en este ámbito, siéndoles dificultoso desarrollar los procesos antes nombrados. Una apropiación deficiente de dicha competencia conduce necesariamente a falencias en la argumentación crítica, la buena comprensión de textos y escritura de los mismos.

\section{Problema investigativo}

El desarrollo de la competencia comunicativa del lenguaje es una de las aptitudes esenciales que debe tener un individuo para que pueda desenvolverse satisfactoriamente en ámbitos académicos, profesionales e incluso interpersonales (Ministerio de Educación Nacional, 2006). Esto, a su vez, se debe a que el lenguaje es una facultad que habilita al ser humano para comunicarse sobre bases conceptuales complejas y multideterminadas, así como niveles altos de abstracción. Se deduce así que, sin un buen uso del lenguaje, el individuo se encuentra alienado de la participación activa en las instituciones sociales que condicionan su vida. Por eso el ser humano debe ser capaz de, no solo de comprender discursos sino también de emitirlos. 


\section{Para 2012, las pruebas PISA indicaban que un $47 \%$ de los estudiantes de 15 años en Colombia no alcanzaba el nivel mínimo de desempeño en la competencia de lectoescritura.}

Es por esto que el desarrollo de las competencias en lectoescritura es uno de los ejes de la educación colombiana (Ministerio de Educación Nacional, 2006) y se manifiesta en la prueba de estado realizada en el nivel superior de la educación, en donde estas competencias se agrupan dentro del componente de lectura crítica. Esta prueba, denominada Saber pro, es aplicada por el Instituto Colombiano para el Fomento de la Educación Superior (ICFES), y tiene como objetivo el "comprobar el grado de desarrollo de las competencias de los estudiantes próximos a culminar los programas académicos de pregrado" y "proporcionar información para la comparación entre programas, instituciones y metodologías, y mostrar su evolución en el tiempo" (Decreto 3963 de 2009).

Sin embargo, el desempeño de los estudiantes de secundaria colombianos en lectura crítica tiende a ser preocupante. Tenemos como ejemplo las pruebas PISA, que para 2012 indicaba que un $47 \%$ de los estudiantes de 15 años en Colombia no alcanzaba el nivel mínimo de desempeño en la competencia de lectoescritura (Semana, 2016). Así, las universidades están llamadas a cumplir un papel fundamental para llenar los vacíos que puedan tener sus estudiantes admitidos en cuanto a estas habilidades.

Es necesario, por lo tanto, un permanente estudio de la pertinencia de la educación superior, la calidad de los programas impartidos y el impacto que tiene sobre sus estudiantes. En este estudio nos centramos principalmente en la relación de dicho impacto con la manera como se comporta la demanda laboral en Colombia. Así, nos preguntamos por el vínculo entre las capacidades de lectoescritura y el nivel de vinculación laboral, que serán estudiados desde los programas de pregrado de la Universidad Nacional, sede Bogotá, evaluando tanto la demanda como el desempeño en esta competencia básica. Con base en estas ideas nos planteamos esta pregunta para guiar la siguiente investigación:

¿Cuál fue el desempeño en lectoescritura de los graduados de la Universidad Nacional de Colombia, sede Bogotá, en los cuatro programas de pregrado con mayor demanda por parte del mercado laboral durante el año 2016 ?

\section{Objetivo general}

Contrastar los resultados del desempeño en lectoescritura de todos los programas de pregrado con los estándares fijados por la prueba
Saber pro, el escalafón de los de mayor acogida laboral y los resultados de las carreras con mejor desempeño.

\section{Objetivos específicos}

Recopilar los resultados para el año 2016-2 de los indicadores antes definidos y evaluar su distribución en relación con el desempeño general de la carrera.
Comparar entre sí todos los programas de pregrado en cuanto al desempeño en lectoescritura.

Establecer la relación entre los programas de mejor desempeño en lectoescritura y aquellos con la mayor demanda por parte del mercado laboral. 


\section{La competencia de lectura crítica}

La lectura crítica es definida por las pruebas de estado como la capacidad que tienen los estudiantes para entender, interpretar y evaluar textos. Esta capacidad se divide en tres niveles de competencias: el primero y más básico es entender los contenidos locales de un texto, los significados de sus elementos y la identificación de eventos explícitos en la narración; la segunda competencia requiere comprender textos de manera global, analizando cada una de sus partes y relacionándolas entre sí; el tercer nivel de competencia requiere establecer una posición crítica frente al texto; para esto se debe reconocer información implícita de él, ser capaz de relacionar los contenidos de este con otros textos y contextualizar la lectura con la época y el lugar en el que se desarrolló. Es en esta competencia donde el estudiante relaciona su valoración crítica frente al mundo con la valoración a la que el texto quiere llevarlo y en la que debe involucrar todo su bagaje social y cultural.

\section{Hipótesis y marco teórico}

La hipótesis que trataremos de corroborar o refutar en este estudio es que:

No hay una relación directa entre la habilidad de lectura crítica de los graduados de las Universidad Nacional (sede Bogotá) con el nivel de demanda del mercado laboral durante el año 2016.

A continuación, profundizaremos sobre las dos variables que conforman nuestra hipótesis operativa.

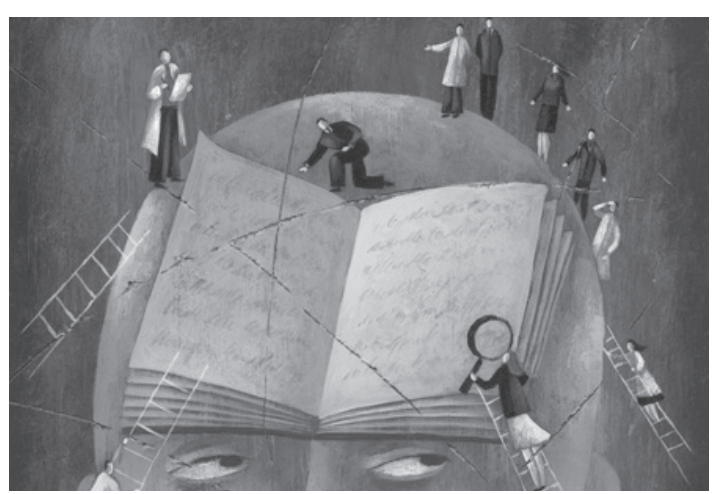

\section{Demanda del mercado laboral}

Es necesario un indicador numérico que cuantifique el nivel de demanda de profesionales recién graduados, y con él, establecer las carreras a analizar con más alto grado de vinculación laboral. El Ministerio de Educación cuenta desde 2007 con el Observatorio Laboral para la educación (OLE), que se encarga producir información estadística detallada sobre la formación académica de los egresados colombianos de las Instituciones de Educación Superior (IES) y su situación laboral (aportes a seguridad social, nivel de ingreso, empleabilidad, vinculación laboral, etc.). El observatorio publica periódicamente información tanto de oferta como de demanda del mercado. Para este trabajo usaremos la información de la segunda, obtenida por la institución a través de encuestas directas a los principales y más representativos empleadores del país, además de estudios de prospectiva sectorial.

La fuente publicó en el año 2016 un documento titulado Perfil Académico Y Condiciones De Empleabilidad: Graduados De Educación Superior (2001-2014) (Observatorio Laboral para la Educación, 2016) que compila y presenta los resultados de trece años del perfil académico y la Encuesta de Seguimiento a Graduados de pregrado. De este recurso se usarán los datos de la sección Vinculación Laboral de los Graduados, que cruza los datos académicos con los del Sistema de Seguridad Social Integral (EPS, ARL y AFP). 
Tabla 1. Vinculación laboral y salario promedio de los graduados de acuerdo al informe del OLE (Observatorio Laboral para la Educación, 2016).

\begin{tabular}{|c|l|c|l|c|}
\hline \multirow{2}{*}{$\begin{array}{c}\text { Posición relativa } \\
\text { (escalafón) }\end{array}$} & \multicolumn{2}{|c|}{ Por vinculación laboral } & \multicolumn{2}{c|}{ Por salario de enganche } \\
\cline { 2 - 5 } & \multicolumn{1}{|c|}{ Programa } & Vinculación & \multicolumn{1}{|c|}{ Programa } & Salario de enganche (COP) \\
\hline 1. & Medicina & $94,2 \%$ & Geología & $\$ 3^{\prime} 327.357$ \\
\hline 2. & Ing. Mecatrónica & $93,5 \%$ & Medicina & $\$ 3^{\prime} 017.286$ \\
\hline 3. & Estadística & $89,2 \%$ & Ing. Mecatrónica & $\$ 3^{\prime} 002.146$ \\
\hline 4. & Geología & $88,9 \%$ & Ing. Minas & $\$ 2^{\prime} 359.685$ \\
\hline 5. & Ing. Minas & $81,9 \%$ & Estadística & $\$ 2^{\prime} 346.300$ \\
\hline- & Media Universitaria & $80,7 \%$ & Media Universitaria & $\$$ 1’736.849 $^{2}$ \\
\hline
\end{tabular}

Se escogen dos indicadores de demanda básicos para trabajar, enganche o vinculación laboral, y salario de enganche. Por vinculación se hará referencia al porcentaje de graduados que consiguieron empleo en el semestre a partir de la obtención de su título profesional, especificando el porcentaje de graduados que realizan cotizaciones a la seguridad social. Entiéndase por salario de enganche la remuneración mensual promedio por la prestación de los servicios a un empleador por los graduados de un programa académico de pregrado. Más adelante se mostrará cómo estos dos indicadores de demanda pueden diferir, aunque no radicalmente, pues miden el nivel de empleabilidad, por un lado, y el de ingreso, por otro. Se seleccionarán los cinco programas presentes en la sede Bogotá de la Universidad Nacional con más altos índices de empleabilidad e ingreso, a través de los indicadores ya descritos.

En la demanda laboral, los datos del informe publicado en abril de 2016 por el OLE muestran en la tabla 1:

De las anteriores carreras, una de ellas no tiene programa de pregrado en la Sede Bogotá de la UN, por tanto, reducimos el estudio a Medicina, Ingeniería Mecatrónica, Estadística y Geología. La diferencia respecto a la media es considerable, con un enganche de hasta $14 \%$ por encima, y en los salarios, en el caso de Geología, de hasta 91,57\% por encima del salario promedio.

\section{Desempeño en lectoescritura}

La segunda variable a evaluar, habiendo seleccionado las carreras por demanda, es la competencia de lectura crítica, medida para los graduados a través de las pruebas de estado Saber Pro. Se compararán los programas

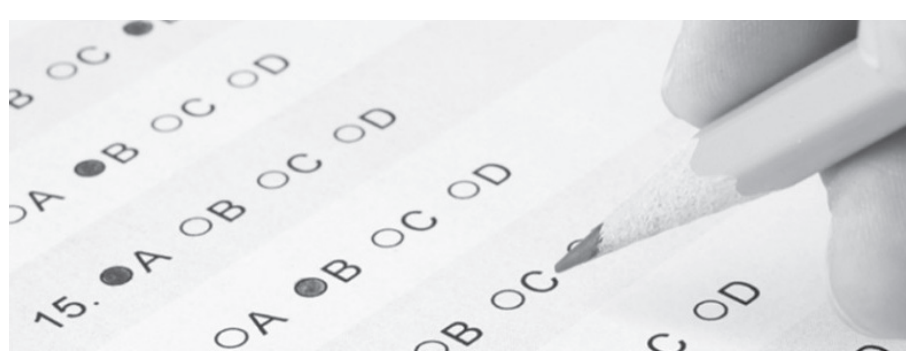

mediante la cantidad de egresados de cada uno que se encuentra en un nivel superior en el componente de lectura crítica. Para esto, se hará uso de la distribución porcentual del desempeño de los estudiantes que presentaron la prueba. El ICFES, en cada uno de sus reportes por carrera, ofrece esta distribución por medio de un gráfico (ej. Fig. 1). Particularmente se hará énfasis en el porcentaje de estudiantes que alcanzan un desempeño por encima del nivel 4. Los 4 niveles de desempeño son definidos por el ICFES como "una descripción cualitativa del desempeño de los estudiantes". 


\section{Metodología}

Planeamos poner a prueba nuestra hipótesis a través del análisis y la interpretación de los resultados que emite el ICFES. Para esto, recurrimos a sus bases de datos, que arrojan resultados agregados (esto es, el promedio aritmético de todos los resultados individuales) a nivel de institución educativa, sede y programa individual. Nuestro trabajo consiste en comparar entre sí todos los programas individuales, enfocándonos en el componente de lectoescritura, para identificar aquellos con mejor desempeño agregado y, evidenciar si coinciden con los que el observatorio nacional identifica como los de mayor acogida en el mercado laboral.

Igualmente compararemos los programas respecto a qué cantidad de egresados de cada uno se encuentran en un nivel superior en el componente de lectura crítica. Para esto, nos guiaremos por la distribución porcentual del desempeño de los estudiantes que presentaron la prueba. El ICFES, en cada uno de sus reportes por carrera, ofrece esta distribución por medio de un gráfico como el de la figura 1. Particularmente nos enfocaremos en el porcentaje de estudiantes que alcanzan un desempeño por encima del nivel 4 .

Se pretende comparar la demanda del mercado laboral (por empleabilidad e ingreso), ordenando programas seleccionados con este indicador y paralelamente mostrar y ordenar por desempeño en lectoescritura, con su respectiva dispersión. La posible concomitancia o las diferencias más relevantes entre ambas medidas permitiría establecer o desmentir relaciones, al menos directas, entre el nivel de demanda laboral y el desempeño en lectoescritura de los egresados de las carreras seleccionadas de la Sede Bogotá de la Universidad Nacional de Colombia.

Decidimos limitarnos solamente a la Universidad Nacional por tres razones. En primer lugar, por viabilidad: muchas instituciones de educación superior del país no cuentan con los mismos programas curriculares por lo que la agregación de los puntajes se tornaría dispendiosa. Por otro lado, por coherencia: la calidad de un mismo programa en varias instituciones podría variar significativamente, dependiendo del énfasis de cada universidad, la antigüedad de la misma y de la facultad en cuestión, entre otros factores. Esto podría distorsionar gravemente nuestros resultados y mermar nuestras conclusiones. Finalmente, elegimos a la Universidad Nacional como objeto de estudio por su amplia gama de programas y su posición líder en formación profesional, lo que nos permitirá derivar conclusiones extrapolables a otros contextos e instituciones educativas.

\section{Resultados}

Tomando los resultados agregados de cada uno de los programas curriculares de pregrado en la Universidad Nacional de Colombia, sede Bogotá, y aislando los resultados de lectura crítica, se encuentra que, como enuncia la hipótesis, los puntajes más altos en este componente no corresponden con los programas de mayor demanda laboral actualmente. Los resultados expresados en la figura
2, que corresponden al puntaje agregado por programa curricular en el componente (color rojo) muestran a las primeras cuatro carreras como Filosofía, Estudios Literarios, Psicología e Historia en este orden. De los 48 programas, Medicina, Ingeniería Mecatrónica, Geología y Estadística se encuentran en los puestos 10, 24, 38 y 41, respectivamente. 
Figura 1. Agregado por programa en lectoescritura

Puntaje agregado

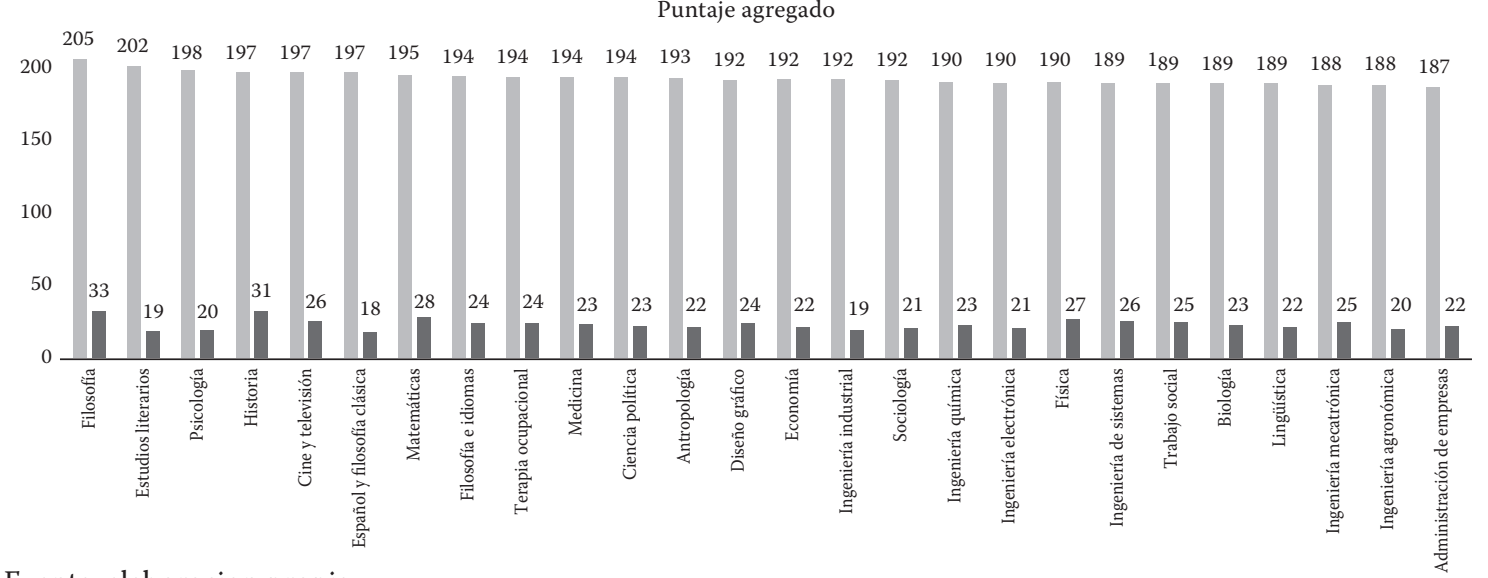

Fuente: elaboracion propia

Figura 2. Agregado por programa en Lectoescritura ${ }^{1}$

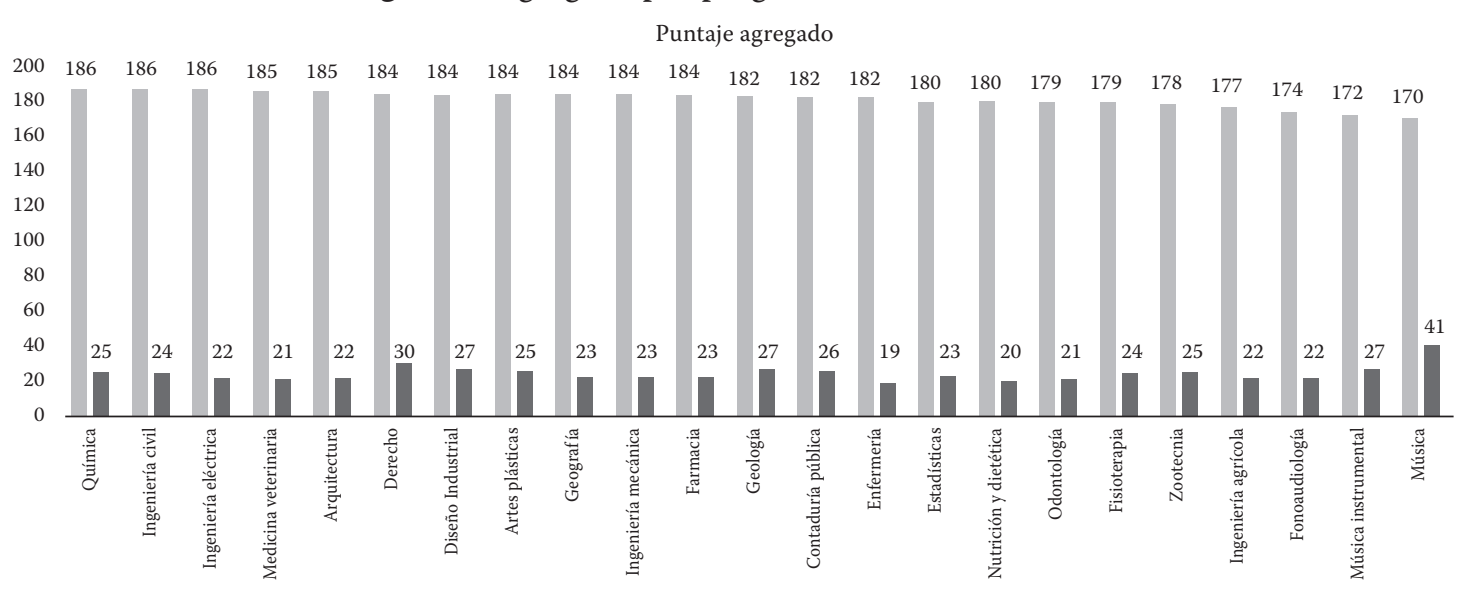

Fuente: elaboración propia

Figura 3. Porcentaje de estudiantes que alcanzaron un desempeño superior Porcentaje por encima de N4

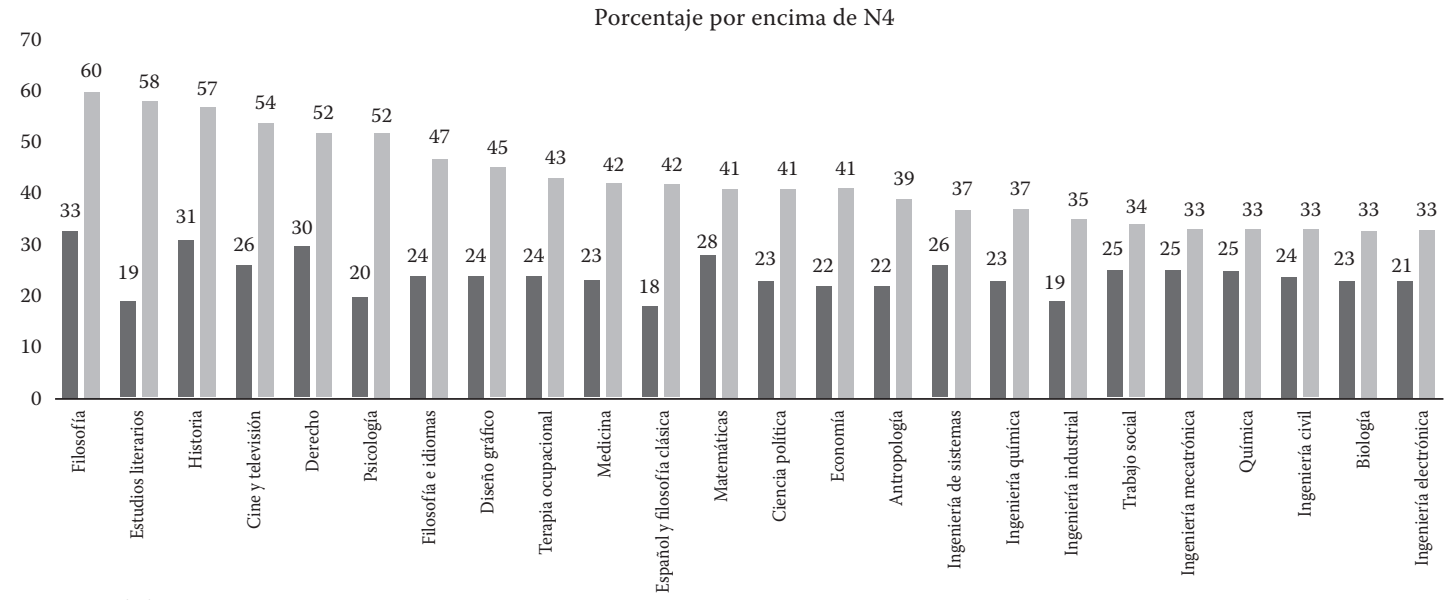

Fuente: elaboración propia

1 Los resultados agregados por programa curricular, institución educativa, sede y a nivel nacional pueden ser consultados en el enlace oficial del ICFES: http:/www2.icfesinteractivo.gov.co/resultados-saber2016-web/ pages/publicacionResultados/agregados/saberPro/consultaAgregadosIES.jsf\#N o-back-button 
Más dicientes aún que los puntajes, son las cifras porcentuales que indican la cantidad de estudiantes que se encuentran por encima del nivel 4 (color amarillo).

Los programas con el mayor número de estudiantes encima del nivel superior coinciden con los de mayor puntaje agregado, a excepción de Psicología, que es reemplazada por un margen de apenas dos puestos por Cine y televisión.

Para evitar reducir el sesgo respecto al análisis de los resultados también tuvimos en cuenta la desviación estándar, es decir, qué tanto se alejan los puntajes más altos y más bajos del promedio agregado en cada programa. En caso de que la dispersión fuera significativamente más alta en los extremos de la gráfica, daría a entender que los resultados agregados extremos se deben en gran medida a que existen estudiantes particulares que se alejan extraordinariamente de la media y distorsionan la visión global del desempeño una vez agregados los puntajes. No obstante, la varianza (de color gris en las figuras 2 y 3 ) no se agrupa de manera particularmente clara en los extremos, con la excepción de los programas de Filosofía, Historia y Música particularmente en la sección de puntajes agregados. Esto quiere decir que los promedios aritméticos guardan un nivel de fidelidad similar con sus programas correspondientes.

\section{Conclusiones}

Luego de haber analizado los resultados podemos afirmar que nuestra hipótesis es cierta. Esto puede en parte deberse al hecho de que las empresas colombianas se enfocan en otras habilidades, por ejemplo; el manejo eficaz del inglés.

Los colombianos se están graduando sin las capacidades que requiere el mercado laboral. "Uno de los ejemplos más claros es el dominio del inglés a un nivel profesional, lo que limita el desarrollo de los profesionales y su posibilidad de obtener mejores empleos y un mayor salario" (Portafolio, 2017).

La brecha entre los trabajadores que sí poseen las habilidades requeridas y los que no la tienen es tan amplia que, al no haber trabajadores calificados, los empleadores encuentran necesario recurrir a trabajadores de otros países para cubrir la demanda laboral.

Las carreras que obtuvieron los puntajes más altos en el componente de lectoescritura son precisamente aquellas que requieren una lectura más rigurosa y periódica que las demás, a saber: Filosofía, Psicología, Estudios literarios e Historia.
El hecho de que dichas carreras hayan, no solo obtenido un puntaje relativamente alto sino mantenido un porcentaje mayor al cincuenta por ciento de estudiantes en nivel 4, habla de una uniformidad positiva en el nivel de sus egresados.

Las carreras que obtuvieron un puntaje menor en lectoescritura son aquellas en las que más que una lectura juiciosa, se requiere de habilidades prácticas que poco tienen que ver con el componente mencionado, a saber: Odontología, Zootecnia, Ingeniería agrícola y Música.

De las anteriores tres premisas se puede concluir algo más general: los egresados no poseen parámetros uniformes en cuanto a su habilidad de lectura crítica, ya que sus áreas de desempeño los llevan a enfocarse casi exclusivamente en sus propias dinámicas académicas.

Las carreras mejor remuneradas y más demandadas en el país son aquellas que requieren un ejercicio cuantitativo-práctico. Esto se debe en parte a la naturaleza industrial de la economía colombiana y cómo su modo de desempeño está más que todo enfocada en la actividad agrícola y minera. 
De las carreras con mayor demanda en el mercado laboral colombiano, aquellas con un mayor desempeño en el componente de lectoescritura en la prueba Saber Pro es Medicina. Esto, puede deberse en parte a que siendo su puntaje de ingreso el segundo más alto - luego de Ingeniería mecatrónica-, los estudiantes de por sí han ingresado a la universidad con

\section{Referencias}

Decreto $\mathrm{N}^{\mathrm{O}}$ 3963. Por el cual se reglamenta el Examen de Estado de Calidad de la Educación Superior. 14 de octubre de 2009. República de Colombia. Disponible en http://www. mineducacion.gov.co/1621/articles-205955_ archivo_pdf_decreto3963.pdf

Ministerio de Educación Nacional. (2008). Estándares Básicos de Competencias en Lenguaje, Matemáticas, Ciencias y Ciudadanas. Bogotá: Ministerio de Educación Nacional.

Observatorio Laboral para la Educación. (abril de 2016). Perfil Académico y Condiciones de Empleabilidad: Graduados de Educación Superior (2001-2014). Obtenido de Observatorio para la Educación Superior: http://www. graduadoscolombia.edu.co/html/1732/articles-348102_recurso_3.pdf un nivel de habilidad de lectura crítica más o menos estandarizado (el examen evalúa un componente completo de dicha habilidad).

Por otro lado, de dichas carreras, la que obtuvo un peor desempeño en el componente fue Estadística. Esto, probablemente, por su enfoque netamente cuantitativo (explicado anteriormente).
Observatorio Laboral para la Educación. (2017). Observatorio Laboral para la Educación. Obtenido de Estructura del sistema de información: http://www.graduadoscolombia. edu.co/html/1732/w3-article-347409.html

Portafolio. (23 de febrero de 2017). ¿Cómo ha cambiado el mercado laboral en Colombia? Obtenido de http://www.portafolio.co/ economia/empleo/mercado-laboral-en-colombia-para-2017-503619

Semana. (12 de marzo de 2016). ¿Por qué los estudiantes colombianos no comprenden lo que leen? Obtenido de http://www.semana.com/ cultura/articulo/pruebas-saber-11-nivel-delectura- sigue-siendo-falencia-del-pais/507868

\section{Anexo 1}

Lista de programas curriculares en la Universidad Nacional de Colombia con su respectivo código de acreditación de alta calidad emitido por el SNIES (Sistema Nacional de Información sobre la Educación Superior)

\begin{tabular}{|l|r|}
\hline Programa Curricular & Código SNIES \\
\hline Facultad de Artes & 30 \\
\hline Arquitectura & 2497 \\
\hline Artes Plásticas & 6 \\
\hline Cine y Televisión & 4 \\
\hline Diseño Gráfico & 5 \\
\hline Diseño Industrial & 3100 \\
\hline Música & 3101 \\
\hline Música Instrumental & \\
\hline Facultad de Ciencias & 31 \\
\hline Biología
\end{tabular}

\begin{tabular}{|l|c|}
\hline Estadística & 32 \\
\hline Farmacia & 37 \\
\hline Física & 33 \\
\hline Geología & 34 \\
\hline Matemáticas & 35 \\
\hline Química & 36 \\
\hline Facultad de Ciencias Agrarias \\
\hline Ingeniería agronómica & 1 \\
\hline Facultad de Ciencias Económicas \\
\hline Administración de Empresas & 19 \\
\hline Contaduría Pública & 16895 \\
\hline
\end{tabular}




\begin{tabular}{|l|r|}
\hline Economía & 18 \\
\hline Facultad de Ciencias Humanas \\
\hline Antropología & 13 \\
\hline Español y Filología Clásica & 54036 \\
\hline Estudios Literarios & 22 \\
\hline Filología e Idiomas & 23 \\
\hline Filosofía & 20 \\
\hline Geografía & 3103 \\
\hline Historia & 21 \\
\hline Lingüística & 16938 \\
\hline Psicología & 14 \\
\hline Sociología & 16 \\
\hline Trabajo Social & 15 \\
\hline Facultad de Derecho, Ciencias Políticas y Sociales \\
\hline
\end{tabular}

\begin{tabular}{|l|r|}
\hline Ciencia Política & 3140 \\
\hline Derecho & 17 \\
\hline Facultad de Enfermería & \\
\hline Enfermería & 7 \\
\hline Facultad de Ingeniería & \\
\hline
\end{tabular}

\begin{tabular}{|l|c|}
\hline Ingeniería Agrícola & 24 \\
\hline Ingeniería Civil & 25 \\
\hline Ingeniería de Sistemas & 26 \\
\hline Ingeniería Eléctrica & 27 \\
\hline Ingeniería Electrónica & 16941 \\
\hline Ingeniería Industrial & 16940 \\
\hline Ingeniería Mecánica & 28 \\
\hline Ingeniería Mecatrónica & 16939 \\
\hline Ingeniería Química & 29 \\
\hline Facultad de Medicina & 8 \\
\hline Fisioterapia & 3102 \\
\hline Fonoaudiología & 9 \\
\hline Medicina & 10 \\
\hline Nutrición y Dietética & 12 \\
\hline Terapia Ocupacional & 2 \\
\hline Facultad de Medicina Veterinaria y Zootecnia \\
\hline Medicina Veterinaria & 3 \\
\hline Zootecnia & 11 \\
\hline Facultad de Odontología & \\
\hline Odontología & \\
\hline
\end{tabular}

\section{Anexo 2.}

Tabla con los resultados oficiales de 2016 emitidos por el ICFES en las pruebas Saber Pro en el componente de lectoescritura. Las convenciones utilizadas son las siguientes:

- Puntaje global: puntaje agregado del desempeño en la totalidad de la prueba (con un máximo de 300 puntos obtenibles).

- Lectura crítica: Puntaje agregado en el componente de lectura crítica (con un máximo de 300 puntos obtenibles).

- Dispersión en el componente: varianza con respecto a la media en el componente de lectura crítica
- Porcentaje por encima de N4: porcentaje de los estudiantes evaluados que se encuentran por encima del nivel superior bajo los estándares del ICFES (expresado en puntos porcentuales)

- Grupo: cifras correspondientes al grupo de referencia del programa curricular. Este grupo está conformado por todos los programas curriculares del país que pertenecen a una misma área. Por ejemplo, el programa de Contaduría pública estará asociado al grupo de administración de empresas y contaduría pública, que reúne a todos los programas de pregrado pertenecientes a esta dependencia a nivel nacional.

\begin{tabular}{|l|c|c|c|c|c|c|c|}
\hline $\begin{array}{c}\text { Programa } \\
\text { Curricular }\end{array}$ & $\begin{array}{c}\text { Puntaje } \\
\text { Global }\end{array}$ & $\begin{array}{c}\text { Lectura } \\
\text { Crítica }\end{array}$ & $\begin{array}{c}\text { Dispersión } \\
\text { en el } \\
\text { componente }\end{array}$ & $\begin{array}{c}\text { Porcentaje } \\
\text { por encima } \\
\text { de No. 4 }\end{array}$ & $\begin{array}{c}\text { Lectura } \\
\text { Crítica } \\
\text { Grupo }\end{array}$ & $\begin{array}{c}\text { Dispersión } \\
\text { Grupo }\end{array}$ & $\begin{array}{c}\text { Porcentaje } \\
\text { Grupo por } \\
\text { encima de No. 4 }\end{array}$ \\
\hline $\begin{array}{l}\text { Administración de } \\
\text { Empresas }\end{array}$ & 185 & 187 & 22 & 29 & 144 & 28 & 3 \\
\hline Antropología & 187 & 193 & 22 & 39 & 158 & 33 & 10 \\
\hline Arquitectura & 183 & 185 & 22 & 24 & 156 & 29 & 6 \\
\hline Artes Plásticas & 177 & 184 & 25 & 25 & 159 & 30 & 8 \\
\hline Biología & 186 & 189 & 23 & 33 & 168 & 29 & 14 \\
\hline Ciencia Política & 186 & 194 & 23 & 41 & 158 & 33 & 10 \\
\hline Cine y Televisión & 190 & 197 & 26 & 54 & 159 & 30 & 8 \\
\hline
\end{tabular}




\begin{tabular}{|c|c|c|c|c|c|c|c|}
\hline $\begin{array}{l}\text { Programa } \\
\text { Curricular }\end{array}$ & $\begin{array}{l}\text { Puntaje } \\
\text { Global }\end{array}$ & $\begin{array}{l}\text { Lectura } \\
\text { Crítica }\end{array}$ & $\begin{array}{l}\text { Dispersión } \\
\text { en el } \\
\text { componente }\end{array}$ & $\begin{array}{c}\text { Porcentaje } \\
\text { por encima } \\
\text { de No. } 4\end{array}$ & $\begin{array}{c}\text { Lectura } \\
\text { Crítica } \\
\text { Grupo }\end{array}$ & $\begin{array}{c}\text { Dispersión } \\
\text { Grupo }\end{array}$ & \begin{tabular}{|c|} 
Porcentaje \\
Grupo por \\
encima de No. 4
\end{tabular} \\
\hline Contaduría Pública & 180 & 182 & 26 & 25 & 141 & 26 & 2 \\
\hline Derecho & 187 & 184 & 30 & 52 & 156 & 30 & 7 \\
\hline Diseño Gráfico & 184 & 192 & 24 & 45 & 159 & 30 & 8 \\
\hline Diseño Industrial & 181 & 184 & 27 & 26 & 159 & 30 & 8 \\
\hline Economía & 191 & 192 & 22 & 41 & 169 & 29 & 14 \\
\hline Enfermería & 175 & 182 & 19 & 16 & 147 & 28 & 3 \\
\hline Español y Filología Clásica & 187 & 197 & 18 & 42 & 173 & 31 & 21 \\
\hline Estadística & 181 & 180 & 23 & 19 & 168 & 29 & 14 \\
\hline Estudios Literarios & 190 & 202 & 19 & 58 & 173 & 31 & 21 \\
\hline Farmacia & 183 & 184 & 23 & 21 & 143 & 29 & 3 \\
\hline Filología e Idiomas & 187 & 194 & 24 & 47 & 142 & 31 & 3 \\
\hline Filosofía & 190 & 205 & 33 & 60 & 173 & 31 & 21 \\
\hline Física & 187 & 190 & 27 & 30 & 168 & 29 & 14 \\
\hline Fisioterapia & 176 & 179 & 24 & 12 & 143 & 29 & 3 \\
\hline Fonoaudiología & 172 & 174 & 22 & 0 & 143 & 29 & 3 \\
\hline Geografía & 180 & 184 & 23 & 25 & 158 & 33 & 10 \\
\hline Geología & 182 & 182 & 27 & 25 & 168 & 29 & 14 \\
\hline Historia & 187 & 197 & 31 & 57 & 173 & 31 & 21 \\
\hline Ingeniería Agrícola & 177 & 177 & 22 & 12 & 155 & 29 & 6 \\
\hline Ingeniería Agronómica & 183 & 188 & 20 & 26 & 150 & 29 & 4 \\
\hline Ingeniería Civil & 186 & 186 & 24 & 33 & 155 & 29 & 6 \\
\hline Ingeniería de Sistemas & 187 & 189 & 26 & 37 & 155 & 29 & 6 \\
\hline Ingeniería Eléctrica & 185 & 186 & 22 & 27 & 155 & 29 & 6 \\
\hline Ingeniería Electrónica & 189 & 190 & 21 & 33 & 155 & 29 & 6 \\
\hline Ingeniería Industrial & 191 & 192 & 19 & 35 & 155 & 29 & 6 \\
\hline Ingeniería Mecánica & 183 & 184 & 23 & 23 & 155 & 29 & 6 \\
\hline Ingeniería Mecatrónica & 189 & 188 & 25 & 33 & 155 & 29 & 6 \\
\hline Ingeniería Química & 191 & 190 & 23 & 37 & 155 & 29 & 6 \\
\hline Lingüística & 179 & 189 & 22 & 24 & 173 & 31 & 21 \\
\hline Matemáticas & 188 & 195 & 28 & 41 & 168 & 29 & 14 \\
\hline Medicina & 188 & 194 & 23 & 42 & 170 & 28 & 14 \\
\hline Medicina Veterinaria & 183 & 185 & 21 & 28 & 150 & 29 & 4 \\
\hline Música & 160 & 170 & 41 & 20 & 159 & 30 & 8 \\
\hline Música Instrumental & 164 & 172 & 27 & 17 & 159 & 30 & 8 \\
\hline Nutrición y Dietética & 176 & 180 & 20 & 16 & 143 & 29 & 3 \\
\hline Odontología & 173 & 179 & 21 & 17 & 143 & 29 & 3 \\
\hline Psicología & 187 & 198 & 20 & 52 & 152 & 29 & 5 \\
\hline Química & 185 & 186 & 25 & 33 & 168 & 29 & 14 \\
\hline Sociología & 184 & 192 & 21 & 32 & 158 & 33 & 10 \\
\hline Terapia Ocupacional & 181 & 194 & 24 & 43 & 143 & 29 & 3 \\
\hline Trabajo Social & 177 & 189 & 25 & 34 & 158 & 33 & 10 \\
\hline Zootecnia & 173 & 178 & 25 & 17 & 150 & 29 & 4 \\
\hline
\end{tabular}

